\title{
Phosphorylation sites of HER2/c-erbB-2: role in cell growth and in disease
}

\author{
Rukhshan Khurshid', Mahjabeen Saleem ${ }^{2}{ }^{\star}$, Gul-e-Raana ${ }^{3}$ and Muhammad Saleem Akhthar ${ }^{2}$ \\ 1Department of Biochemistry, Fatima Jinnah Medical College, Lahore, Pakistan; ${ }^{2}$ nnstitute of Biochemistry and Biotechnology, University of the \\ Punjab, Lahore, Pakistan; ${ }^{3}$ Department of Biochemistry, Gujranwala Medical College, Gujranwala, Pakistan
}

The protein kinase c-erbB-2 belongs to the family of receptor tyrosine kinase and is involved in oncogenesis. The present study predicts different phosphorylation sites of HER2/c-erbB-2 which are important in preventing or developing cancer, especially breast cancer. Sequence homology showed highest homology (77\%) with epidermal growth factor receptor kinase domain. According to PROSITE search result, active sites of c-erbB-2 are $\mathrm{N}$-lobe (glycine rich phosphate binding loop). Catalytic loop with presumptive catalytically active of Asp108 is phosphorylated by tyrosine protein kinase. A-loop, activation loop, becomes phosphorylated and activates the substrate binding. The study strengthens our knowledge regarding HER2 signaling by the detection of uncharacterized signaling proteins, establishing phosphorylation of an activation loop and helps us to make assumptions about the role of such previously unidentified proteins. On the basis of importance of HER2 in breast cancer as well as in other diseases, this study provides fruitful information for designing new therapeutic strategies.

Key word: phosphorylation sites, HER2/c-erbB-2, cell growth and disease

Received: 06 June, 2013; revised: 09 October, 2014; accepted: 30 October, 2014; available on-line: 14 November, 2014

\section{INTRODUCTION}

The c-erbB-2 gene is also called HER-2/neu because of its association with neural tumors. Its gene product is a $185 \mathrm{kDa}$ (p185HER) transmembrane protein expressed on epithelial cells and related to the epidermal growth factor receptor (Cho et al., 2003).

Expression of c-erbB-2 is found in individuals with breast, ovarian and gastrointestinal tumors. In breast cancer, the gene is a useful prognostic predictor of the general survival and also a period of relapse in breast cancer patients (Slamon et al., 1987). Elevated serum cerbB-2 antigen levels showed correlation with decreased response to hormone therapy for breast cancer. Of the three oncogenes, c-erbB-2, ras and c-myc, c-erbB-2 has the strongest prognostic value in breast cancer (Cho et al., 2003).

The protein kinase c-erbB-2 belongs to the receptor tyrosine kinase (RTK) family. Members of RTK include cerbB-1, 2, 3 and 4 (HER2, HER3 and HER4), epidermal growth factor and transforming growth factor. Members of this family are frequently hyperactive in solid tumors. Many RTKs are involved in oncogenesis because of the mutation or chromosome translocation or by gene overexpression. This may results in hyperactive kinase that confers an anomalous, ligand-independent, unregulated growth impetus to the cancerous cells (Rowinsky, 2004).

There are thousands distinct protein phosphorylation sites in a cell. It is estimated that in some cellular state one tenth to one half of proteins are phosphorylated. Phosphorylation at tyrosine residues affects a wide range of properties in proteins such as enzyme activity, subcellular localization, and interaction between molecules. Furthermore, tyrosine kinases play a substantial role in many signal transduction cascades and gene expression (Radhakrishnan et al., 2012). According to Hunter (1998) phosphorylated tyrosine amino acid residues are recognized by specific binding domains of other proteins. Therefore, these types of interactions are involved in triggering various intracellular signaling pathways. However, as a result of mutations, some tyrosine kinase enzymes become constitutively active which is an uninterrupted functional state that might produce impact on the initiation or progression of cancer (Hunter, 1998; Radhakrishan et al., 2012).

All members of RTK require phosphorylation for full activity. Signaling, which induces ligand oligomerization, is a major function of this family in normal cell. These RTKs are essential components of signal transduction pathways that affect cell proliferation etc. (Stamos et al., 2002). RTKs are single transmembrane receptors that possess intrinsic cytoplasmic enzymatic activity, catalyzing the transfer of the phosphate group from ATP to tyrosine residues in protein substrates. The phosphotyrosine residues either enhance receptor catalytic activity or provide docking sites for downstream signaling proteins (Hubbard, 1999).

Reversible phosphorylation produces a conformational change in the structure of many enzymes and receptors, causing them to become activated or deactivated (van Wareen et al., 1998). It is generally accepted that reversible phosphorylation of protein tyrosine residues is a signaling mechanism implicated in cell proliferation, adhesion, differentiation, transformation and apoptosis (Monteiro \& Stern, 1996). It also controls gene transcription, synaptic transmission and insulin action. Phosphorylation of proteins by protein tyrosine kinase adds phosphate to specific tyrosine in target proteins; phosphate is removed from phosphorylated tyrosine by enzymes called protein

e-mail: mahjabeensaleem1@hotmail.com

Abbreviations: HER2, human epidermal growth factor receptor 2; erbB-2, avian erythroblastosis oncogene B 2; RTK, receptor tyrosine kinase; EGFR, epidermal Growth Factor Receptor; EGFRK, epidermal Growth Factor Receptor kinase; CK2, casein kinase 2; PKC, protein kinase $\mathrm{C}$ 
tyrosine phosphatases. This phosphatase may be capable of dephosphorylating critical substrates involved in transformation processes (Roskoski, 2005).

The phosphorylation is controlled by the opposing actions of protein tyrosine kinase and protein tyrosine phosphatases (Monteiro \& Stern, 1996; Bixby, 2001). An imbalance between these enzymes may impair normal cell growth leading to cellular transformation (Sun \& Tonks, 1994). Oncogenic activation of tyrosine kinases results in the constitutive activation of a conditionally regulated enzyme activity (Rodriguez \& Park, 1994). Aberrant tyrosine phosphorylation is a hallmark of many types of cancer and other human diseases (Hunter, 1998).

Present study predicts the structure of HER-2/neu and possible phosphorylation sites in the structure. We have predicted how these phosphorylation sites are important in preventing or developing the cancer, especially breast cancer. We hypothesize that phosphorylated erbB2 may provide either more significant or additional prognostic marker data cancer patients.

\section{MATERIALS AND METHODS}

Sequence analysis. c-erbB-2 consists of 293 amino acids. Primary sequence of c-erbB-2 was retrieved from SWISSPROT data bank (Bairoch \& Apweiler, 1998) using the basic BLAST algorithim (Vouzis \& Sahinidis, 2011). Sequence homology searches of the Protein Data Bank (PDB), using the basic BLAST algorithm were carried out independently for each primary sequence.

Model building and assessment. The automated homology model building was performed using the protein structure modeling program MODELLER (Sali \& Blundell, 1993). Reliability of the predicted homology model was assessed by the ENERGY command of the MODELLER. Evaluation was further conducted by PROCHECK (Laskowski et al., 1993) and WHATCHECK (Altschul et al., 1990).

\section{RESULTS AND DISCUSSION}

As a result of dimerization of HER2, autophosphorylation of tyrosines takes place in the cytoplasmic tail of the receptors and elicits a variety of signaling pathways i.e. promote cell proliferation and opposes apoptosis and therefore, prevents uncontrolled cell growth (Olayioye, 2001).

The homology model was assessed stereo chemically by PROCHECK. The plot shows that $91.8 \%$ of the residues lie in the core, $7.8 \%$ in the allowed region and $0.4 \%$ in the generously allowed region. No residue lies in disallowed region. The superposition of the C-alpha backbone of c-erbB-2 and respective template was also detected and it was observed that the structurally conserved regions in the CrebB, the corresponding SCR of template with the greatest sequence similarity is superposed on the frame work.

\section{Active sites of c-erbB-2}

According to PROSITE search results, N-lobe is a glycine rich phosphate binding loop consisting of amino acid residues GIWIPDG at position 1-7. Catalytic loop consists of a motif of 11 amino acid residues (RLVHRDLAARN) present at 104-114 with plausible catalytically active Asp108. Catalytic loop is phosphorylated by tyrosine protein kinase. C-lobe consists of a motif of 22 amino acid residues (DVRLVHRDLAARN-
VLVKSPNHV) present at position 102-123. The A-loop (Asp127-Val148) is the activation loop which is phosphorylated and activates the substrate binding.

\section{Important elements of catalytic machinery of c-erbB-2}

Like EGFR kinase domain (template of c-erbB-2), $\mathrm{N}$ terminal lobe mainly consists of $\beta$ strands and one $\alpha$ helix, while larger $C$ terminal lobe is mostly $\alpha$ helices containing motif, and a cleft separates these structures. The probable catalytic residue is Asp809 in EGFRK (Asp109 in c-erbB-2). The catalytic loop comprises His812-Arg818 in EGFRK (His107-Arg113 in c-erbB-2) while A-loop amino acid residues ranges Asp831-Val852 (Asp127-Val148 in c-erbB-2).

\section{Phosphorylation sites}

\section{$\mathrm{N}$-terminal lobe}

Like EGFR (Gly695-Gly700), the predicted model of c-erbB-2 has a glycine rich nucleotide phosphate binding loop, Gly1 to Gly7 (GIWIPDG). This loop shows two hydrogen bonds: between Ile12 $\rightarrow$ Trp3 and Val14 $\rightarrow$ Gly1. Results show the presence of non covalent interactions between 2 highly conserved side chains in the $\mathrm{N}$ lobe of active kinase enzyme that interact with phosphate group of ATP or its analogue. In c-erbB-2, there are 2 salt bridges between Lys17 $\rightarrow$ Glu34 and Lys17 $\rightarrow$ Asp127.

We observed that Glu34 is the site of tyrosine kinase phosphorylation (Table 1). From PROSITE results, a patch of amino acids from 29-36 (KEIIDEAY) is observed in predicted structure. Kulathu and Komander (2012) have observed that this site may regulate cell function. Another phosphorylation site is protein kinase C (PKC) phosphorylation site, Ser24-Lys26 and it is reported by Wolf-Yadlin et al. (2006) that the phosphorylation of HER2 by PKC stimulates the cell proliferation process in normal cells. Over expression of HER2/neu signaling may induce the expression of specific mRNAs, which contribute to the increased metastatic potential of HER2/neu overexpressing cancer cells (Kapler, 2000; Wolf-Yadlin et al., 2006).

Baselga (2006) reported that in EGFR, Met742 and Cys 751 are the interaction sites for the inhibitor, whereas Gln767 and Met769 also participate in kinase inhibitor complex formation. In the predicted model, there is Met38 and instead of Cys there is Ser47. According to WHATIF, the surface accessibility of both Met38 and Ser47 is near to zero. So it means that these amino acids are in buried state or present near the core/catalytic site and may have the ability to contact substrate or inhibitor. We also observed Gln63 (Gln767) and Met65 (Met769) in the predicted structure and that Gln63 has high surface accessibility (18.64 $\AA 2)$ but the surface accessibility of Met65 is near to zero. This shows that Gln63 may interact with other amino acids present near the surface

Table 1. H-bonding pattern in calcium binding domain Asp276(O) $\rightarrow($ OE2)Glu274

Asp277(N) $\rightarrow($ OD1)Asp276

Asp283(O) $\rightarrow(\mathrm{N})$ Glu286

Ala284(O) $\rightarrow(\mathrm{N})$ Tyr287

Glu285(OE2) $\rightarrow($ OD1)Asp265 
and Met65 may have the ability to form complexes with the substrate or inhibitor.

Klingbell and Gill (1999) have reported conserved basic residues in kinase enzyme family specific for tyrosine kinase activity. They described that Arg779, Lys782 and Lys855 in EGFR provide positively charged area to the surface of the molecule. They also observed a mutation of Lys855 to Ala responsible for destabilization and partial inactivation of enzyme. Arg779 mutation had little effect on the $\mathrm{Km}$ value for peptide substrate though change of Lys782 increased the $\mathrm{Km}$ value for ATP fold representing its role in ATP binding. Therefore, it is proposed that Lys782 may be one of the residues containing ribose binding site of epidermal growth factor receptor. In our study, in the predicted structure of c-erbB-2, Arg75 is present in the equivalent position of $\operatorname{Arg} 779$, Lys151 at position of Lys782 and Arg78 at position of Lys855. Thus, it is possible that Arg 78 may play a role in ATP binding and also a ribose binding site of c-erbB-2. In the predicted structure, oxygen atom of Arg78 shows hydrogen bonding with nitrogen atom of Lys81. Its NE shows hydrogen bonding with $\mathrm{O}$ atom of Phe182. On the other hand, Arg75 forms three ion pairs with Asp72 and Lys151 forms two salt bridges with Asp188. Its NZ atom forms hydrogen bond with oxygen of Tyr187. Surface accessibility of $\operatorname{Arg} 75$ and $\operatorname{Arg} 78$ is $24.84 \AA 2$ and $37.46 \AA 2$, respectively. It provides an area of positive charge to the surface of the molecule, a property that all members of kinase enzyme family share. The angle between N-lobe and C-lobe of kinase structure was smaller and brings important catalytic element into vicinity.

\section{Catalytic site}

A-loop mainly consists of loop-beta-loop-beta-loop (Fig. 1). The activation loop is located at the tip of the active site between the $\mathrm{N}$-terminal and the C-terminal lobes of the kinase domain. In kinase regulation, it usually serves as a switch between an unphosphorylated (closed conformation) and a phosphorylated (open conformation) that blocks and supports substrate binding, respectively (Gotoh et al., 1992).

The well conserved sequence of the EGFRK catalytic loop (HRDLAARN) is also present in insulin receptor kinase, sharing structure with these RTKs. In EGFR, the catalytic loop constitutes Asp812-Asn818, the presumptive catalytic residue is Asp813 (general base) and the Aloop ranges from Asp831-Val852. On the other hand in c-erbB-2, the catalytic loop constitutes Arg107-Asn114. According to PROSITE results, the possible protein kinase tyrosine phosphorylation site is 105Leu-117Val (LVHRDLAARMVLV). This region also comprises a

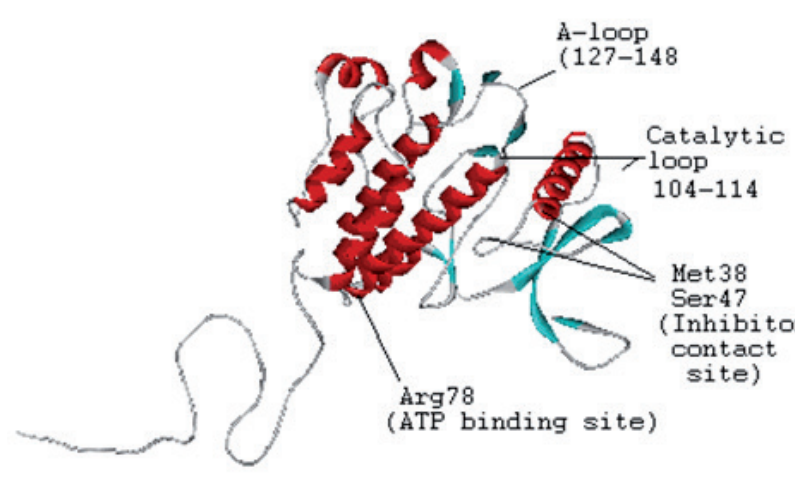

Figure 1. Functional sites of HER2 catalytic loop, His107-Asn114 (HRDLAARN). Arg108 is present in the catalytic loop and has tyrosine protein kinase specific activity. In RTKs, the phosphorylation in the A-loop causes it to undergo a large structural reorganization that releases steric or chemical constrictions on the catalytic site and produces optimal activity (Hubbard \& Till, 2000).

\section{A-loop}

In most protein kinases, the activation loop (A-loop) assumes its catalytic conformation after phosphorylation of a tyrosine or threonine amino acid residue. In EGFR, the activation loop tyrosine residue (Tyr877) is phosphorylated and its phosphorylation status correlates with its activity level in intact cells (Mukherje et al., 2006). Another study reported that in HER2, Y877 (Y172) located in the activation loop of the kinase domain, may be associated with regulation of HER2 signaling (Bose et al., 2006). In our predicted structure, the casein kinase II phosphorylation site was observed at Tyr175 (Table 1, Fig. 1). On the other hand, there are other protein kinases that do not require phosphorylation in their Aloop for biological activity i.e. glycogen phosphorylase kinase, casein kinase I etc. (Malecka, 2013).

In the A-loop of EGFRK, there is DFG loop which is important for ATP coordination. In the predicted structure, we also observed the DFG loop (127-129) with very low surface accessibility, and that in the vicinity of DFG loop there is Asn114 (ND2) that forms H-bond with oxygen and OD2 of Asp108 (a catalytic amino acid). H-bonding is observed between the amino acids of A-loop region and the amino acids present in the vicinity (Table 2). Among these, the most important amino acid is Arg108 (a catalytic amino acid) which forms H-bond with Leu130. This may link the region of catalytic site with A-loop. This site also has tyrosine sulfation site, which is required for maximal catalytic activity and according to Hoffhiness and coworkers (2009) sulfation plays a central role in enhancing protein-protein interaction. Other important amino acids are Arg161, Arg162 and Phe163 having cAMP phosphorylation sites. On the other hand, Arg104 is present near Leu105 (protein kinase tyrosine phosphoryation site). It is reported that oncoprotein HER-2/neu exhibiting tyrosine kinase activity causes phosphorylation and activation of many second-messenger systems and that tyrosine kinase signal transduction pathways are becoming imperative targets for therapeutic interventions (Luftner et al., 2003). Wong and coworker (2009) have described that level of tyrosine-phosphorylation was greatly increased in the HER-2/ neu positive breast tumors, therefore displaying its asso-

Table 2. H-bonding pattern of amino acids of A-loop region

\begin{tabular}{lc}
\hline H-bonding (A-loop) & Surface accessibility \\
\hline Arg108(NH1) $\rightarrow(\mathrm{O})$ Leu130 & 5.76 \\
\hline Leu134(N) $\rightarrow(\mathrm{O})$ Arg104 & 1.74 \\
\hline Asp135(O) $\rightarrow(\mathrm{NH} 2)$ Arg104 & 24.59 \\
\hline Ile136(O) $\rightarrow(\mathrm{NH} 1)$ Arg104 & 36.85 \\
\hline Glu140 $(\mathrm{OE} 2) \rightarrow(\mathrm{NH} 1)$ Arg162 & 9.17 \\
\hline Glu140 $(\mathrm{OE} 1) \rightarrow(\mathrm{NH} 1)$ Arg162 & \\
\hline Tyr141 $(\mathrm{N}) \rightarrow(\mathrm{O})$ Phe163 & 11.72 \\
\hline Ala143 $(\mathrm{N}) \rightarrow(\mathrm{O})$ Arg161 & 0.69 \\
\hline Arg161 $(\mathrm{NH} 1) \rightarrow(\mathrm{O})$ Asp144 & 14.80 \\
\hline
\end{tabular}




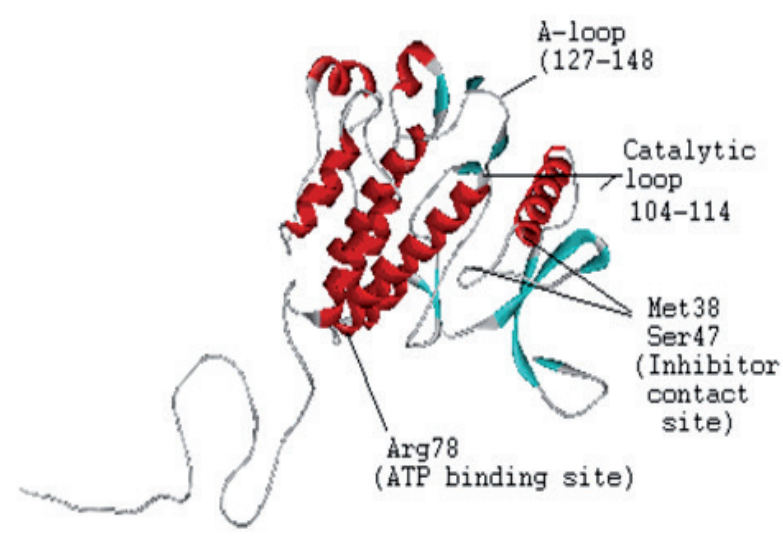

Figure 2. Phosphorylation sites of HER2

ciation with tumor progression as well as demonstrating its potential as prognostic marker.

The presence of 4 Glu residues in the A-loop of EGFRK has been suggested to be responsible for intrinsic catalytic activity. In our predicted model there are only 2 Glu residues i.e. Glu138 and Glu140 present in the loop.

\section{C-terminal lobe}

Autophosphorylation of key tyrosine residue in the C-terminal portion of receptor lead to subsequent signal transduction. Next, the phosphorylated tail recruits other signaling molecules and transmits the signal to downstream pathways (Boss \& Zhang, 2009). The regulatory function of C-terminal tail of EGFR was suggested as a result of various deletion mutations at the C-terminal domain, which can increase autophosphorylation and/or transforming activity of the receptor (Chang et al., 1997; Boss \& Zhang, 2009).

\section{Intramolecular contact of the extreme carboxyl terminus}

\section{Calcium binding domain}

In EGFR, there are 19 residues (Leu977-Pro995) of C-terminal region which are highly acidic (LMDEEDMDDVVDADEYLIP). In the predicted structure of cerbB-2, this acidic region is from Leu273-Pro290 (LEDDDMGDLVDAEEYLVP). According to PROSITE, a calcium binding domain is present in this region i.e. from Asp275-Tyr287 which is highly acidic. H-bonding pattern of amino acid residues of this domain is given in Table 1, Fig. 2. It is observed that except Lys281 all amino acids have the surface accessibility in a range of 12-34 $\AA 2$. The signaling for regulation of metabolic processes is initiated by binding of a ligand (e.g. EGF) to the extracellular region of these receptors, which then transduce the signal to the intracellular region through single transmembrane helix. Then, the intracellular tyrosine kinase domain phosphorylates several tyrosine residues in the C-terminal tail of the receptor (Schlessinger, 2002).

\section{Casein kinase 2 (CK2) phosphorylation}

CK2, a holoenzyme containing two catalytic (CK2 $\alpha$ or $\mathrm{CK} 2 \alpha^{\prime}$ ) and two regulatory (CK2 $\beta$ ) subunits, is a constitutive enzyme that is ubiquitously expressed in cytoplasm as well as in the nucleus of eukaryotic cells (Fig. 2). It is observed that many primary breast tumor tissue samples and human cancer cells display an increase in either CK2 or IKK activity (Romieu-Mourez et al., 2001). C-terminal region also have casein kinase 2 phosphorylation sites (Table 1). CK2 plays an important role in HER2/neu cell signaling process. However, elevated CK2 activity play a role in aberrant activation of factors responsible for cell signaling and the activation of transcriptional activity of Adaptor-associated kinase (Luo et al., 2003) and it may represents a potential therapeutic target (Ren et al., 2011). Present study found a CK2 phosphorylation site in the C-terminal region. According to PROSITE search results, there are 4 sites i.e. Ser98 (SYLE), Thr175 (TVWE), Ser262 (DPLD) and Ser271 (SLLE) (Table 1).

CAMP and CGMP dependent protein kinase phosphorylation

A cAMP and cGMP dependent protein kinase phosphorylation site is also observed i.e. Arg161 to Tyr164 (Fig. 2). It is reported that this phosphorylation was enhanced by calcium and calmodulin, which plays an important role in calcium transport. All 4 human ErbB kinases have a Tyr residue in their activation loop. This showed that ErbB kinases might use activation loop phosphorylation as a regulatory mechanism (Bose \& Zhang, 2009). It is reported that various deletion mutations at the C-terminal tail may increase autophosphorylation and/or transforming activity of the receptor (Kosoy \& O’ Connell, 2008).

\section{Protein kinase C (PKC) phosphorylation}

Another phosphorylation site is protein kinase C (PKC) phosphorylation site (Ser24-Lys26) (Table 3, Fig. 2). It is reported that the phosphorylation of HER2 by PKC stimulate the cell proliferation process in normal cell. Tyrosine kinase phosphorylation sites in HER 2 help in regulating many indispensable cellular functions (Schulze et al., 2005).

It is reported that the proximal polypeptide region is coupled to the C-lobe by the completely buried Leu955 side chain and strongly suggested that the Leu955 side chain does not interact with other proteins (Stammos et al., 2002), on the contrary in our predicted structure, there is Val251 instead of Leu955. The $\mathrm{N}$ atom of Val251 forms H-bond with oxygen atom of Pro247. Val251 has an accessibility of $28.60 \AA 2$. Therefore, it is possible that unlike EGFR, c-erb-2 due to its Val251 interacts with other proteins or receptors and provides a site for subsequent signal transduction. C-lobe of EGFRK also contains the distinctive three amino acid residues i.e. Leu955, Val956 and Ile957 (LVI motif). These amino acids regulate the transphosphorylation of sub-

Table 3. PROSITE Results

CAMP_PHOSPO_site

RRFT 161

PKC_PHOSPO_site

$\mathrm{SPK}^{-} 24$

TYROSINE KINASE_PHOSPHORYLATION_ sites 29-36 KEIIDEAY

$\begin{array}{lr}\text { CK2_PHOSPHO_site } & \\ \text { SYLE } & 98 \\ \text { TVWE } & 175 \\ \text { SPLD } & 262 \\ \text { SLLE } & 271\end{array}$

EF HAND 1

DDDMGDL̄VDAEEY 275

PROTEIN_KINASE_Tyr 105 LVHRDLAARMVLV 
strate tyrosine in oligomerized EGFR family complex in a poorly understood manner. Schaefer and coworkers (1999) have proposed direct contact between this segment and another protein for c-erbB-2/c-erbB-3 system. In our predicted structure, at this position instead of LVF there is VVI (from 251-253) and in this motif, only Val252 shows high surface accessibility (28.60 2 2). On the other hand, $\mathrm{N}$ atom of Val251 forms hydrogen bond with $\mathrm{O}$ of Pro247. The $\mathrm{N}$ atom of Ile253 forms hydrogen bond with OD1 of Asn255.

It is suggested that erbB-2 is a 'ligandless' receptor that is constitutively 'poised' due to its exposed dimerization arm either homodimerize constitutively or heterodimerize with other erbB receptors following their binding to related ligands (Chang et al., 1997).

\section{CONCLUSION}

Epidermal growth factor receptor (EGFR) and its family members, erbB-2, erB-3 and erB-4 are receptor tyrosine kinases which send signals into the cell to regulate many important processes including development, tissue homeostasis, and tumorigenesis. During signaling, the intracellular kinase domain of these receptors is activated by ligand-induced dimerization of the receptor and phosphorylates several tyrosine residues in the C-terminal tail. The phosphorylated tail then recruits other signaling molecules and relays the signal to downstream pathways. This study strengthens the knowledge of HER2 signaling by recognizing previously uncharacterized downstream signaling proteins, establishing phosphorylation of activation loop in HER2, and using network modeling programs to make assumptions about the role of several previously unidentified proteins. Based on critical role of HER2 in breast cancer and other diseases, this study offers appreciated clues for manipulating future therapies.

\section{REFERENCES}

Altschul SF, Gish W, Miller W Myers EW, Lipman DJ (1990) Basic local alignment search tool. J Mol Biol 215: 403-410.

Bairoch A, Apweiler R (1997) The SWISS-PROT protein sequence data bank and its supplement TrEMBL. Nucleic Acids Res 25: 31-36.

Baselga J (2006) Targeting tyrosine kinases in cancer: the second wave. Science 312: 1175-1178.

Bixby JL (2001) Ligands and signaling through receptor type tyrosine phosphatases. IUBMB Life 51: 157-163.

Bose R, Molina H, Patterson AS, Bitok JK, Periaswamy B, Bader JS, Pandey A, Cole PA (2006) Phosphoproteomic analysis of Her2/neu signaling and inhibition. Proc Natl Acad Sci 103: 9773-9778.

Bose R, Zhang X (2009) The ErbB kinase domain: Structural perspectives into kinase activation and inhibition. Exp Cell Res 315: 649658.

Chang H, Riese DJ, Gilbert W, Stern DF, McMahan UJ (1997) Ligands for ErbB-family receptors encoded by a neuregulin-like gene. Nature 387: 509-512.

Cho HS, Mason K, Ramyar KX, Stanley AM, Gabelli SB, Denney Jr DW, Leahy DJ (2003) Structure of the extracellular region of HER2 alone and in complex with the Herceptin Fab. Nature 421: 756-760.

Gotoh N, Tojo A, Hino M, Yazaki Y, Shibuya M (1992) A highly conserved tyrosine residue at codon 845 within the kinase domain is not required for the transforming activity of human epidermal growth factor receptor. Biochem Biophys Res Commun 186: 768-774.

Hoffhines AJ, Jen CH, Leary JA, Moore KL (2009) Tyrosylprotein sulfotransferase-2 expression is required for sulfation of RNase 9 and Mfge8 in vivo. I Biol Chem 284: 3096-3105.

Hubbard SR (1999) Structural analysis of receptor tyrosine kinases. Prog Biophys Mol Biol 71: 343-358.

Hubbard SR, Till JH (2000) Protein tyrosine kinase structure and function. Ann Rev Biochem 69: 373-398.

Hunter T (1998) The phosphorylation of proteins on tyrosine: its role in cell growth and disease. Philos Trans R Soc Lond B Biol Sci 353: 583-605.
Klapper LN, Kirschbaum M.H, Sela M, Yarden Y (2000) Biochemical and clinical implications of the ErbB/HER signaling network of growth factor receptors. Adv Cancer Res 77: 25-79.

Klingbeil CK, Gill GN (1999) A basic residue, Lys 782, composes part of the ATP-binding site on the epidermal growth factor receptor tyrosine kinase. Arch Biochem Biophys 363: 27-32.

Kosoy A, O'Connell MJ (2008) Regulation of Chk1 by its C-terminal domain. Mol Biol Cell 19: 4546-4553.

Kulathu Y, Komander D (2012) A typical ubiquitylation - the unexplored world of polyubiquitin beyond Lys48 and Lys63 linkages. Nature Rev Mol Cell Biol 13: 508-520.

Laskowski RA, McAurthur MW, Moss DS, Thornton JM (1993) PROCHECK: a program to check the stereochemical quality of protein structures. I Appl Crystallogar 26: 283-291.

Lüftner D, Mazurek S, Henschke P, Mesterharm J, Schildhauer S, Geppert R, Wernecke KD, Possinger K (2003) Plasma levels of HER-2/neu, tumor type M2 pyruvate kinase and its tyrosine-phosphorylated metabolite in advanced breast cancer. Anticancer Res 23: 991-997.

Luo T, Lee Y, Saint-Jeannet J, Sargent TD (2003) Induction of neural crest in Xenopus by transcription factor AP2. PNAS 100: 532-537.

Malecka KA (2013) Something from nothing: The first phosphorylation. J Postdoc Res 1: 41-55.

Monteiro HP, Stern A (1996) Redox Modulation of tyrosine phosphorylation dependent signal transduction pathways. Free Radic Biol Med 21: $323-333$.

Mukherji M, Brill LM, Ficarro SB, Hampton G, Schultz PG (2006) A phosphoproteomic analysis of the ErbB2 receptor tyrosine kinase signaling pathways. Biochem-US 45: 15529-15540.

Olayioye MA (2001). Update on HER-2 as a target for cancer therapy: Intracellular signaling pathways of ErbB2/HER-2 and family members. Breast Cancer Res 3: 385-389.

Radahkrishnan SS, Blalock TD, Robinosn PM, Secker G, Daniels J, Grotendorst GR, Schultz GS (2012) Effect of connective tissue growth factor on protein kinase expression and activity in human corneal fibroblasts. Invest Ophthalmol Vis Sci 53: 8076-8085.

Ren K, Xiang S, Zhang W, Ding X, Wu Y, Yang L, Zhou J, Gao X, Zhang J (2011) CK2 phosphorylates AP- $2 \alpha$ and increases its transcriptional activity. BMB Reports 44: 490-495.

Rodriguez CA, Park M (1994) Oncogenic activation of tyrosine kinases. Curr Opin Genet Dev 4: 15-24.

Romieu-Mourez R, Landesman-Bollag E, Seldin DC (2002) Protein kinase CK2 promotes aberrant activation of nuclear factor-kappaB, transformed phenotype, and survival of breast cancer cells. Cancer Res 62: 6770-6778.

Roskoski R (2005) Src kinase regulation by phosphorylation and dephosphorylation. Biochem Biophys Res Comm 331: 1-14.

Rowinsky EK (2004) The erbB Family: targets for therapeutic development against cancer and therapeutic strategies using monoclonal antibodies and tyrosine kinase inhibitors. Ann Rev Med 55: 433-457.

Sali A, Blundell TL (1993) Comparative protein modeling by satisfaction of spatial restraints. I Mol Biol 234: 779-815.

Schlessinger J, Lemmon MA (2003) SH2 and PTB domains in tyrosine kinase signaling. Sci STKE 191: 112.

Schulze WX, Deng L, Mann M (2005) Phosphotyrosine interactome of the ErbB-receptor kinase family. Mol Syst Biol 1: 2005.0008.

Schaefer G, Akita RW, Sliwkowski MX (1999) A discrete three-amino acid segment (LVI) at the C-terminal end of kinase-impaired ErbB3 is required for transactivation of ErbB2. I Biol Chem 274: 859-866.

Slamon DJ, Clark GM, Wong SG, Levin WJ, Ullrich A, McGuire WL (1987) Human breast cancer: correlation of relapse and survival with amplification of the HER-2/neu oncogene. Science 235: 177-182.

Stamos J, Sliwkowiski MX, Eigenbrot C (2002) Structure of the epidermal growth factor receptor kinase domain alone and in complex with a 4-anilinoquinazoline inhibitor. J Biol Chem 277: 46265-46272.

Sun H, Tonks NK (1994) The coordinated action of protein tyrosine phosphatase and kinases in cell signaling. Trends Biochem Sci 19: 480-485.

van Weeren PC, De Bruyn KM, De Vries-Smits AM, van Lint J, Burgering BM (1998). Essential role for protein kinase B (PKB) in insulin-induced glycogen synthase kinase 3 inactivation. Characterization of dominant-negative mutant of PKB. J Biol Chem 273: 13150-13160.

Vouzis PD, Sahinidis NV (2011) GPU-BLAST: using graphics processors to accelerate protein sequence alignment. Bioinformatics 27: 182-188.

Wolf-Yadlin A, Kumar N, Zhang Y, Hautaniemi S, Zaman M, Kim HD, Grantcharova V, Lauffenburger DA, White FM (2006) Effects of HER2 overexpression on cell signaling networks governing proliferation and migration. Mol Syst Biol 2: 54.

Wong LL, Chang CaF, Koay ESC, Zhang D (2009) Tyrosine phosphorylation of $\mathrm{PP} 2 \mathrm{~A}$ is regulated by HER-2 signalling and correlates with breast cancer progression. Int J Oncol 34: 1291-1301. 